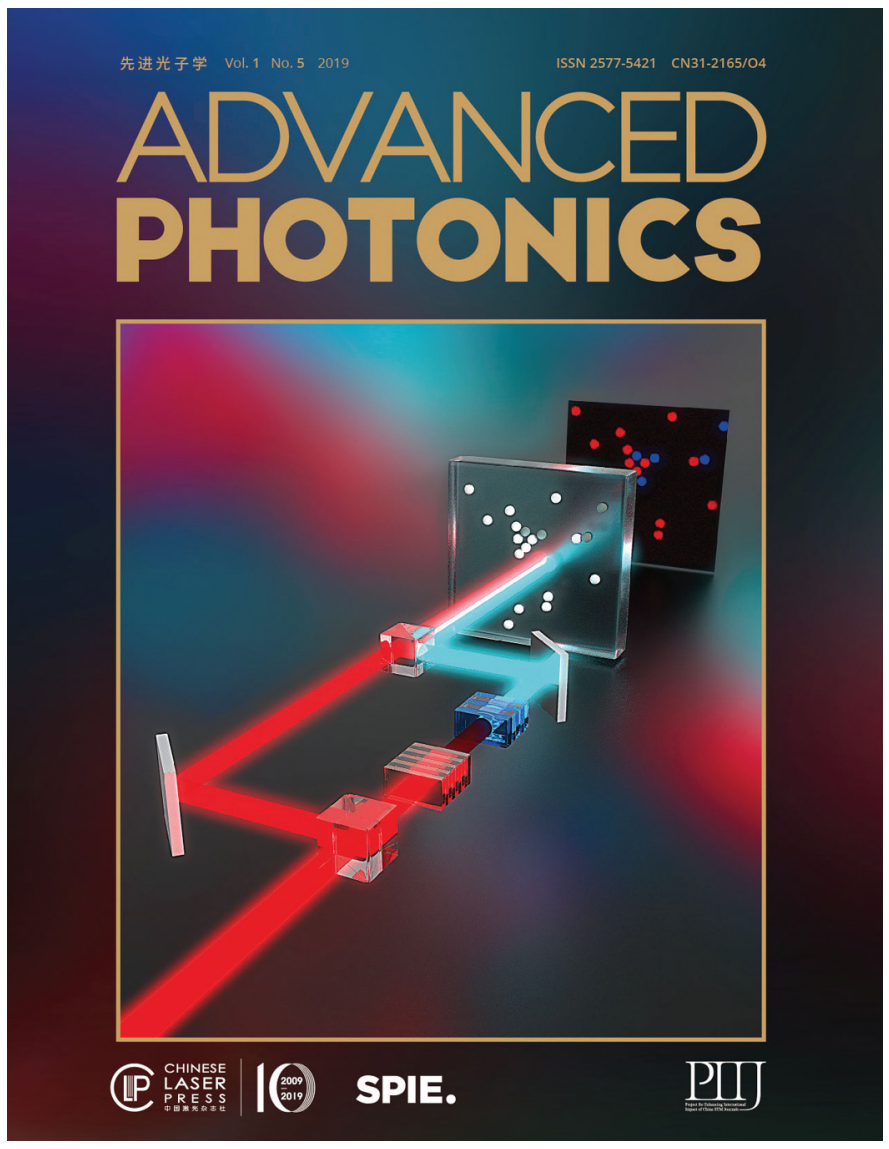

\title{
About the cover: Advanced Photonics Volume 1, Issue 5
}

The image on the cover for Advanced Photonics Volume 1, Issue 5 , is an artistic rendering of a novel multicolor laser light source that allows for chemical-selective imaging in SRS and CARS microscopy with low-intensity noise (image credit: Sterltech Optics). The fully-automated, rapidly tunable laser system is presented in the Advanced Photonics Letter authored by Heiko Linnenbank, Tobias Steinle, Florian Mörz, Moritz Flöss, Han Cui, Andrew Glidle, and Harald Giessen, "Robust and rapidly tunable light source for SRS/CARS microscopy with low-intensity noise," Adv. Phot. 1(5), 055001, doi: 10.1117/1.AP.1.5.055001. 